This item was submitted to Loughborough's Research Repository by the author.

Items in Figshare are protected by copyright, with all rights reserved, unless otherwise indicated.

\title{
High order disturbance observer design for linear and nonlinear systems
}

PLEASE CITE THE PUBLISHED VERSION

http://dx.doi.org/10.1109/ICInfA.2015.7279597

PUBLISHER

(c) IEEE

VERSION

AM (Accepted Manuscript)

LICENCE

CC BY-NC-ND 4.0

REPOSITORY RECORD

Su, Jinya, Wen-Hua Chen, and Baibing Li. 2016. "High Order Disturbance Observer Design for Linear and Nonlinear Systems". figshare. https://hdl.handle.net/2134/21204. 


\section{High Order Disturbance Observer Design for Linear and Nonlinear Systems*}

\author{
Jinya Su and Wen-Hua Chen \\ Department of Aeronautical and Automotive Engineering \\ Loughborough University \\ Loughborough, LE11 3TU, U.K. \\ \{J.Su2,W.Chen\}@lboro.ac.uk
}

\author{
Baibing Li \\ School of Business \& Economics \\ Loughborough University \\ Loughborough, LE11 3TU, U.K. \\ B.Li2@lboro.ac.uk
}

\begin{abstract}
In this paper, a disturbance observer is proposed for nonlinear systems with high order disturbance, where not only disturbance but also its high order derivatives are estimated. The relationship of the proposed observer with the existing results is discussed. Then, the result is further extended to the case of minimal-order output-based disturbance observer design for linear systems subject to high order disturbances. Two practical examples about actuator fault diagnosis for a nonlinear missile system and disturbance estimation for a double-effect pilot plant evaporator system with unobservable states are provided to illustrate the effectiveness of the proposed approaches.
\end{abstract}

Index Terms - Disturbance observer, Fault diagnosis, High order disturbance, Minimal order, Nonlinear systems.

\section{INTRODUCTION}

Disturbance observers (DOs) have received considerable attention in the past three decades due to their versatile applications in the fields of disturbance rejection control [1-5], fault diagnosis [6-8], etc. In classical DO design (see, $[1,2]$ among many others), disturbances are usually assumed to be unknown constants due to the fact that little prior information [9] on disturbances may be available. As a result, the disturbance estimation accuracy and consequently disturbance rejection performance may not be satisfactory in the presence of more complicated disturbances and parameter uncertainties.

To this end, there is a trend in DO design to take the disturbance model information into account. There are two approaches available to model unknown disturbances. The first one is using a neutrally stable exogenous system to represent non-vanishing disturbances (see, [10] for output regulation theory, and $[4,11]$ for DO based control (DOBC) theory). Although step disturbance and periodic disturbances with known period can be represented by neutrally stable exosystems, general disturbances can not be generated. The other approach is using a time series expansion (or polynomial) to approximately represent the disturbance (see, [12-14]

\footnotetext{
*This work is jointly funded by UK Engineering and Physical Sciences Research Council (EPSRC) and BAE Systems under grant (EP/H501401/1). This paper has been accepted by 2015 IEEE International Conference on Information and Automation, August 8-10, Lijiang, China. This is a personal copy from Jinya Su. For simulation study, contact Jinya Su at J.Su2@lboro.ac.uk.
}

among many others), based on which a so-called High Order Disturbance Observer (HODO) can be designed. This paper focuses on the latter disturbance modelling approach for high order disturbance estimation.

The concept of HODO was originally proposed in frequency domain [12] and further discussed in recent literature [15] for robust stability analysis. In those papers, the high order derivatives of disturbances are assumed to be limited, based on which the DOs are designed using the $Q$-filter approach. Although the frequency-domain approach has multiple merits including being concept-simple and suitable with standard transfer function analysis tools, the disadvantages, as highlighted in [13], are that they can only be applied to a class of linear systems and unable to deal with transient performance. To this end, $[6,13,14,16-18]$ considered the HODO problem using the state-space approach. In [16], the concept of proportional multiple-integral observer (PMIO) was used to obtain the state estimate, while [6] considered the problem of simultaneous state and disturbance/fault estimation for linear descriptor systems, which can be seen as the full-order HODO for linear descriptor system. [17] considers the problem of state and disturbance estimation for linear systems based on the concept of generalized extended state observer (ESO) and later [18] shows that this approach can obtain better control performance than the classical ESO [19] as demonstrated by a DC motor control experiment due to the fact that a better disturbance estimation can be achieved by taking more disturbance model information into account.

Recently, Kim et al [13] pioneered HODO design for nonlinear systems assuming that all the states are directly measurable. Then, they further extended the results to minimalorder output-based constant disturbance estimation for linear systems. Since then, this approach has received considerable attention and has been applied in many control applications such as Permanent-Magnet Stepper Motors control [20], output regulation [21], etc. However, there are still two remaining problems in this promising approach. First, the information of the derivative and higher order derivatives of the disturbances can not be obtained in [13] and these information is indispensable in mismatched disturbance rejection control (see, [22, 23], etc.) Secondly, the minimal-order output-based disturbance observer for linear systems in [13] can only 
effectively handle constant disturbances rather than general high order disturbances due to the specific structure of the nonlinear DO, i.e., involving multiple integral action.

This paper addresses the aforementioned two problems. First, for a general nonlinear system with high order disturbances, a HODO is designed assuming all the system states are directly measurable. Second, the relationship of the proposed HODO with the existing results is discussed. Then, the result is further extended to the case of minimal order output based HODO design for linear systems subject to high order disturbances. Finally, the actuator fault diagnosis for a nonlinear missile system and disturbance estimation for a linear double-effect pilot plant evaporator with unobservable states are used to evaluate the proposed HODO for both linear and nonlinear systems.

\section{Problem statement}

This paper considers the nonlinear systems with disturbance,

$$
\dot{x}=f(x, u ; t)+g(x) d,
$$

where $x \in R^{n}, u \in R^{m}, d \in R, f(\cdot), g(x)$ are known nolinear functions, $u$ is the control inputs and $d$ denotes the lumped system disturbances, which may include parameter uncertainties, external disturbances and system faults [24]. Following [2,13], all the system states are assumed to be directly measurable.

Following previous study on the topic of HODO design in both frequency domain [12] and time-domain [13], the disturbance is supposed to have a bounded $r^{t h}$ derivative,

$$
\left|d^{(r)}\right| \leq \mu,
$$

where $\mu \in R$ is a bounded constant.

The objective is to estimate the disturbance $d$ and its derivative and higher order derivatives under the disturbance assumption (2) for general nonlinear system (1).

\section{HODO FOR NONLINEAR SYSTEM}

In this section, a nonlinear DO for high order disturbance estimation will be investigated, which can be seen as the extension of the existing results in [2, 4]. It is divided into three parts including disturbance modelling, observer structure and observer gain design.

\section{A. Disturbance modelling}

To fully take the disturbance model into account when designing the DO, a linear model is used to represent the disturbance under the disturbance assumption (2), given by

$$
\left\{\begin{array}{l}
\dot{\omega}=S \omega+D d^{(r)}, \\
d=L \omega,
\end{array}\right.
$$

where the system matrices $S, D, L$ have the following specific structures.

$$
\begin{aligned}
S & =\left[\begin{array}{cc}
O_{(r-1) \times 1} & I_{r-1} \\
0 & O_{1 \times(r-1)}
\end{array}\right], D=\left[\begin{array}{c}
O_{(r-1) \times 1} \\
1
\end{array}\right], \\
L & =\left[1, O_{1 \times(r-1)}\right] .
\end{aligned}
$$

Remark 1: The disturbance $d$ generated by system (3) can accurately represent polynomial disturbances, such as constant, ramp, parabolic disturbance, etc. $[12,13]$. It can also approximately represent other time-varying disturbances with bounded approximation error, where the estimation error is reflected by the bounded $d^{(r)}$ in (3).

\section{B. HODO design}

Inspired by the nonlinear DO theory [2, 4], the HODO is designed in this section based on reduced-order observer theory, which can also explain the disturbance observer in $[2,4]$ using a new perspective. We first combine the state system (1) and the disturbance system (3) such that an extended system can be obtained, given by

$$
\left\{\begin{array}{l}
\dot{x}=f(x, u ; t)+g(x) L \omega, \\
\dot{\omega}=S \omega+D d^{(r)}
\end{array}\right.
$$

The philosophy of reduced-order observer is to directly estimate the unmeasurable extended states using the measurable states. However, to obtain a good disturbance estimation performance, both the state model and disturbance model should be taken into account. To this end, we derive a new reduced-order extended state dynamic to be estimated. Define a nonlinear invertible transformation with $p(x)$ to be determined later

$$
z=\left[\begin{array}{l}
z_{1} \\
z_{2}
\end{array}\right]=\left[\begin{array}{c}
x \\
-p(x)+\omega
\end{array}\right],
$$

Then the dynamics of $z$ can be obtained, given by

$$
\begin{gathered}
\dot{z}_{1}=f\left(z_{1}, u ; t\right)+g\left(z_{1}\right) L\left[z_{2}+p\left(z_{1}\right)\right], \\
\dot{z}_{2}=-\frac{\partial p\left(z_{1}\right)}{\partial z_{1}}\left\{f\left(z_{1}, u ; t\right)+g\left(z_{1}\right) L\left[z_{2}+p\left(z_{1}\right)\right]\right\} \\
+S\left[z_{2}+p\left(z_{1}\right)\right]+D d^{(r)} .
\end{gathered}
$$

Define $l\left(z_{1}\right)=\frac{\partial p\left(z_{1}\right)}{\partial z_{1}}$, then Eq. (6) is equivalently represented by,

$$
\begin{aligned}
\dot{z}_{2}=[ & \left.S-l\left(z_{1}\right) g\left(z_{1}\right) L\right] z_{2} \\
& +\underbrace{S p\left(z_{1}\right)-l\left(z_{1}\right)\left[f\left(z_{1}, u ; t\right)+g\left(z_{1}\right) L p\left(z_{1}\right)\right]}_{T_{k}}+D d^{(r)},
\end{aligned}
$$

where the known term $T_{k}$ can be seen as the lumped known input to $z_{2}$ dynamic.

An observer estimating $z_{2}$ can be designed for system (7),

$$
\dot{\hat{z}}_{2}=\left[S-l\left(z_{1}\right) g\left(z_{1}\right) L\right] \hat{z}_{2}+T_{k},
$$

where $l\left(z_{1}\right)$ is designed such that $S-l\left(z_{1}\right) g\left(z_{1}\right) L$ is asymptotically stable. When $\hat{z}_{2}$ is available, we can obtain the estimate of $\omega$ and consequently $\hat{d}$ and $\hat{d}^{(i)}$, given by

$$
\left\{\begin{array}{l}
\hat{\omega}=\hat{z}_{2}+p\left(z_{1}\right), \\
\hat{d}=L \hat{\omega} \\
\hat{d}^{(i)}=L_{i} \hat{w}, \text { with } L_{i}=[0, \cdots, \underbrace{1}_{i^{t h}}, \cdots, 0] .
\end{array}\right.
$$

The estimation error dynamic $e_{\omega}=\omega-\hat{\omega}$ is governed by

$$
\dot{e}_{\omega}=\left(S-l\left(z_{1}\right) g\left(z_{1}\right) L\right) e_{\omega}+D d^{(r)} .
$$


Remark 2: Comparing the disturbance estimation error dynamic (10) with that of $[2,4]$, we can obtain the following conclusions. Firstly, if an accurate disturbance model is available (for example neutrally stable model in [4]), we can obtain an exponentially asymptotic estimate of the disturbance. Secondly, when the disturbance model information is not available (such as in [2] and this paper), an approximated disturbance model can still be used (bounded derivative in [2] and bounded high order derivative in this paper) to obtain the disturbance estimate possibly with small estimation error.

\section{The design of $p(x)$}

The general design procedure of $p(x)$ has been proposed in [4]. However, due to the specific structures of the matrices $S$ and $L$ in (4), we can design $p(x)$ using the following procedure.

From the error (10) in conjunction with the definition of $l\left(z_{1}\right)=\partial p\left(z_{1}\right) / \partial z_{1}$, the design of $p(x)$ starts with the design of $l\left(z_{1}\right)$. We define an intermediate variable $\bar{l}\left(z_{1}\right)$ of full row rank such that $l\left(z_{1}\right)=l^{*} \bar{l}\left(z_{1}\right)$ and $\bar{l}\left(z_{1}\right) g\left(z_{1}\right)=I$. Then, (10) reduces to

$$
\dot{e}_{\omega}=(\underbrace{S-l^{*} L}_{\bar{S}}) e_{\omega}+D d^{(r)} .
$$

Due to the specific structure of $S$ and $L$, i.e., the pair $(S, L)$ is in observability canonical form, we can design $l^{*}$ as

$$
l^{*}=\left[\begin{array}{lll}
l_{1}, & \cdots, & l_{r}
\end{array}\right]^{T},
$$

such that the solutions of $P(s):=s^{r}+l_{1} s^{r-1}+\cdots+l_{r-1} s+$ $l_{r}=0$ lie in the left half-plane (LHP). When $l^{*}$ and so $l\left(z_{1}\right)$ are available, we can obtain $p(x)$ based on the relationship $l\left(z_{1}\right)=\partial p\left(z_{1}\right) / \partial z_{1}$.

We further analyse the effect of design parameters on the disturbance estimation performance. Firstly, the system (11) is a bounded-input-bounded-output (BIBO) system due to the stability of $\bar{S}:=S-l^{*} L$, which means the disturbance estimation error $e_{w}$ is always bounded in the presence of bounded $d^{(r)}$. We then derive the transfer function from $d^{(r)}$ to $e_{\omega}$. Without loss of generality, we only consider the transfer function from $d^{(r)}$ to the disturbance estimation error $e_{\omega 1}$, i.e., the first element of $e_{\omega}$, which can be obtained from the following linear system

$$
\left\{\begin{array}{l}
\dot{e}_{\omega}=\bar{S} e_{\omega}+D d^{(r)} \\
e_{\omega 1}=L e_{\omega}
\end{array}\right.
$$

The transfer function from $d^{(r)}$ to $e_{\omega 1}$ is given by

$$
G(s)=L(s I-\bar{S})^{-1} D=\frac{\operatorname{Ladj}(s I-\bar{S}) D}{\operatorname{det}(s I-\bar{S})} .
$$

Using the property

$$
\operatorname{Ladj}(s I-\bar{S}) D=\operatorname{det}\left(\left[\begin{array}{cc}
s I-\bar{S} & D \\
-L & 0
\end{array}\right]\right),
$$

Eq. (14) can be calculated as

$$
G(s)=\frac{1}{s^{r}+l_{1} s^{r-1}+\cdots+l_{r-1} s+l_{r}},
$$

which means the disturbance estimation performance can be exactly analysed using the transfer function (15) and the steady-state disturbance estimation error is given by $e_{s s}=$ $d^{(r)} / l_{r}$.

To further simplify the observer gain design and facilitate the analysis of the relationship of observer order $r$ and disturbance estimation performance, the polynomial $P(s)$ can be chosen in a special form $P(s)=(s+p)^{r}$, where $p$ can be seen as the observer bandwidth. Then the transfer function (15) reduces to:

$$
G(s)=1 /(s+p)^{r},
$$

with the steady-state disturbance estimation error

$$
e_{s s}=d^{(r)} / p^{r} .
$$

That means: ( $i$ ) when the observer order $r$ is fixed, the disturbance estimation error upper bound monotonously decreases with the observer bandwidth p; (ii) an observer with a higher order does not necessarily result in a smaller estimation error and the observer order $r$ should be chosen such that $d^{(r)} / p^{r}$ is as small as possible.

\section{RELATIONSHIP WITH THE EXISTING RESULTS}

In this section, the relationship of the proposed HODO with the existing results will be discussed including the linear case in [14] and the nonlinear cases in $[2,13]$.

\section{A. Relationship with linear case in [14]}

In [14], a HODO is designed for linear system under the assumption that all the states are directly measurable. We will show that it is actually a linear version of the proposed HODO. Without loss of generality, a second order HODO in [14] for a simple system $\dot{x}_{1}=u+d$ is considered, where the observer takes the following form:

$$
\left\{\begin{array}{l}
\hat{d}=p_{1}+l_{1} x_{1}, \\
\dot{p}_{1}=-l_{1}(u+\hat{d})+\hat{\dot{d}}, \\
\hat{\dot{d}}=p_{2}+l_{2} x_{1}, \\
\dot{p}_{2}=-l_{2}(u+\hat{d}),
\end{array}\right.
$$

where $l_{i} s$ are designed such that the solutions of the polynomial $s^{2}+l_{1} s+l_{2}$ are in LHP.

Substituting $\hat{d}$ and $\hat{\dot{d}}$ into the dynamics of $p_{1}$ and $p_{2}$, (17) can be put into an equivalent form, given by

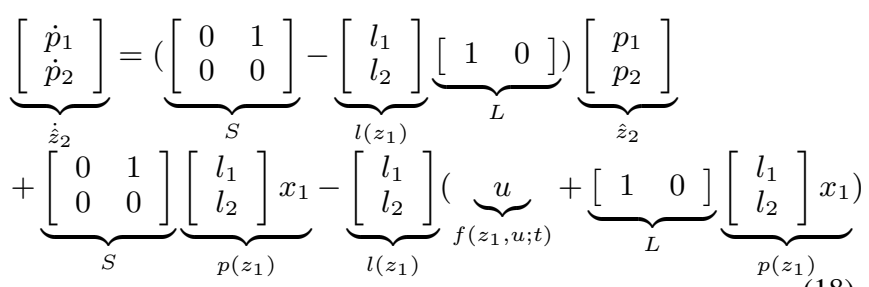


The estimate of $d$ and $\dot{d}$ can be put into the following form

$$
\left\{\begin{array}{l}
\underbrace{\left[\begin{array}{c}
\hat{d} \\
\hat{\dot{d}}
\end{array}\right]}_{\hat{\omega}}=\underbrace{\left[\begin{array}{l}
p_{1} \\
p_{2}
\end{array}\right]}_{\hat{z}_{2}}+\underbrace{\left[\begin{array}{l}
l_{1} \\
l_{2}
\end{array}\right] x_{1}}_{p\left(z_{1}\right)}, \\
\hat{d}=\underbrace{\left[\begin{array}{ll}
1 & 0
\end{array}\right]}_{L} \hat{\omega} ; \hat{\dot{d}}=\underbrace{\left[\begin{array}{ll}
0 & 1
\end{array}\right]}_{L_{1}} \hat{\omega} .
\end{array}\right.
$$

Comparing (18) and (19) with (8) and (9), we can see that the HODO in [14] is the linear version of the proposed HODO with $l\left(z_{1}\right)$ being selected as constant gain $l\left(z_{1}\right)=\left[l_{1}, l_{2}\right]^{T}$.

\section{B. Relationship with nonlinear cases}

In [2], a DO was proposed for general nonlienar system with constant disturbance, which is equivalent to the firstorder HODO proposed in this paper. The paper can be seen as its extension to the case of high order disturbance estimation. Besides, the paper also presents a reduced-order observer perspective to the DO in [2].

In [13], a DO is proposed to estimate higher order disturbances in the time series expansion for nonlinear system. However, the approach proposed in this paper is quite different from that of [13]. Firstly, [13] focuses on obtaining the estimate of disturbance, this paper focuses on obtaining the estimate of disturbance and its high order derivatives. Secondly, the proposed approach can be easily extended to the case of minimal-order output-based high order disturbance estimation for linear systems (as shown in Section V), which can not be easily achieved by the approach in [13] due to the presence of integral terms in the observer structure. Thirdly, the order of the proposed HODO is higher than that of [13], since the information of the high order derivatives of the disturbance is involved in derivation of the disturbance estimate.

\section{EXTENSION TO OUTPUT-BASED HODO}

Consider a linear system with disturbances

$$
\left\{\begin{array}{l}
\dot{x}=A x+B u+D d \\
y=C x
\end{array}\right.
$$

where the disturbances $d$ are of multiple dimension and each element of it is supposed to satisfy (2). An extended system including the state dynamics (20) and disturbance dynamic (3) can be obtained as follows:

$$
\left\{\begin{array}{l}
{\left[\begin{array}{l}
\dot{x} \\
\dot{\omega}
\end{array}\right]=\left[\begin{array}{cc}
A & D L \\
O & S
\end{array}\right]\left[\begin{array}{l}
x \\
\omega
\end{array}\right]+\left[\begin{array}{l}
B \\
0
\end{array}\right] u+\left[\begin{array}{l}
O \\
D
\end{array}\right] d^{(r)},} \\
y=\left[\begin{array}{ll}
C & O
\end{array}\right]\left[\begin{array}{l}
x \\
\omega
\end{array}\right]
\end{array}\right.
$$

Then we can obtain the estimate of $\omega$ using the Luenberger observer theory under the observable condition (see, $[17,19]$ ), which can be seen as the full order HODO, since all the states are involved in derivation of the disturbance $\omega$. However, when the observability of the extended system is not satisfied, this approach can not be directly applied.

In addition, for the purpose of fast disturbance estimation, especially fault estimation in the field of fault diagnosis [7], an observer with lower dimension is more desirable since low computation power and short computation time are required. To this end, there is no need simultaneously estimating the whole extended state vector but only the disturbances vector $d$. Unfortunately, in most cases, to obtain the disturbance estimate, part of the original state vector $x$ has to been estimated such that the existence condition of the observer is satisfied.

We transform the minimal order disturbance estimation problem into functional observer design problem and so the existing tool in [25] can be used. To this end, we define a functional matrix with specific structure

$$
L=\left[\begin{array}{cc}
L_{0} & O \\
O & I
\end{array}\right], \text { and } \bar{x}=\left[x^{T}, \omega^{T}\right]^{T},
$$

where $L_{0}$ is a matrix to be designed. To obtain the estimate of $\omega$, only $L \bar{x}$ rather than $\bar{x}$ are needed to be estimated. So the problem of HODO has been transformed into the problem of functional observer design, i.e., estimating $L \bar{x}$. Its existence condition and the design process can be solved using the tools in [25]. Besides, further detailed discussion on this topic will be provided in the future work.

Remark 3: Since in functional observer based HODO, not all the states $x$ are needed to be estimated in derivation of the disturbance estimate, the existence condition of the proposed HODO can be more easily satisfied compared with that of the classical generalized ESO [17, 18], where the necessary condition of it is that the pair $(A, C)$ is observable.

\section{Simulation STUdY}

In this section, application examples of actuator fault diagnosis for a nonlinear missile system and disturbance estimation for linear unobservable double-effect pilot plant evaporator system are given to illustrate the effectiveness of the proposed approach.

\section{A. Nonlinear case: missile actuator fault diagnosis}

In this subsection, the problem of actuator fault diagnosis through fault estimation approach [7] for a nonlinear missile system is considered. Following [3,7], the longitudinal dynamic of a missile with actuator fault $f_{a}$ is given by

$$
\dot{x}=f(x)+g_{1}(x) u+g_{2}(x) f_{a},
$$

where $x=[\alpha ; q ; \delta]$ denote the angle of attack (degrees), pitch rate (degrees per second) and tail fin defection (degrees). The definition of $f(x), g_{1}(x), g_{2}(x)$ and normal control input $u$ are referred to [3]. We can see that the non-linear missile system (22) falls into the nonlinear system structure (1) and so the HODO can be designed using (8) and (9).

A fault profile is designed in Fig. 1 (real line), which includes step fault (1-3 sec), ramp fault (3-5 sec), second-order polynomial fault (5-7 sec) and sine function fault $2 \sin (2 \mathrm{t})$ (7$10 \mathrm{sec})$. Three kinds of HODO with different observer order are compared including first-order HODO (the same as [2]), second-order and third-order HODO. The observer gain $l(x)$ 

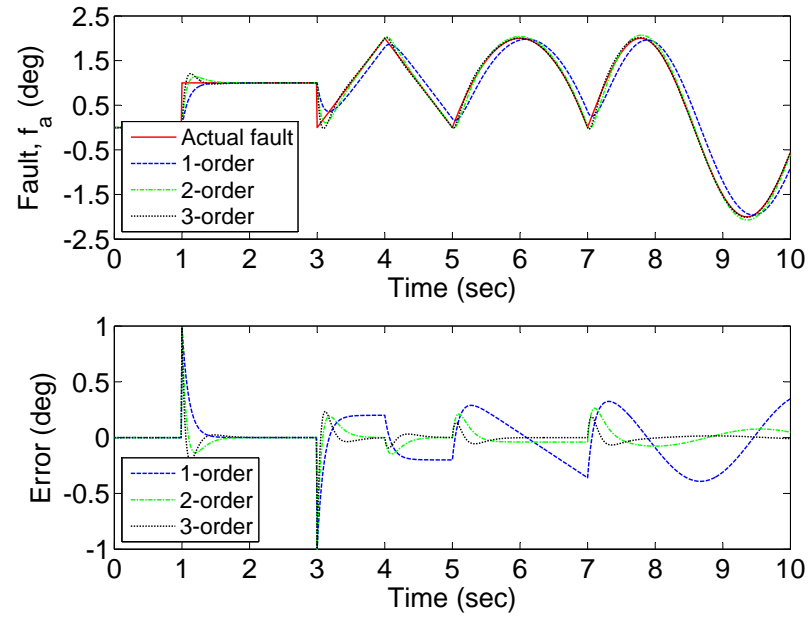

Fig. 1

FAULT ESTIMATE USING HODOS WITH THREE DIFFERENT KINDS OF OBSERVER ORDER.

and the nonlinear function $p(x)$ are designed such that the $p$ in (16) is 10 and are given as follows respectively:

$$
\begin{aligned}
& l_{1}(x)=\left[\begin{array}{lll}
0 & -0.0763 & 0
\end{array}\right], \quad p_{1}(x)=-0.0763 q ; \\
& l_{2}(x)=\left[\begin{array}{lll}
0 & -0.1526 & 0 \\
0 & -0.7628 & 0
\end{array}\right], \quad p_{2}(x)=\left[\begin{array}{l}
-0.1526 q \\
-0.7628 q
\end{array}\right] \text {; } \\
& l_{3}(x)=\left[\begin{array}{lll}
0 & -0.2288 & 0 \\
0 & -2.2885 & 0 \\
0 & -7.6283 & 0
\end{array}\right], \quad p_{3}(x)=\left[\begin{array}{l}
-0.2288 q \\
-2.2885 q \\
-7.6283 q
\end{array}\right] \text {. }
\end{aligned}
$$

The initial states of the observers are supposed to be zero and the simulation results are shown in Fig. 1, where the upper plots depict the fault estimate performance (real red line for actuator fault, blue dash line (first-order HODO), green dash dotted line (second-order HODO), dark dotted line (thirdorder HODO) fault estimate) and the lower plot show the corresponding fault estimation error.

We can see from Fig. 1 that: i) the first-order HODO (the same as that of [2]) can exponentially estimate constant disturbance but is subjected to estimate error for disturbances with non-zero derivative; ii) the second-order HODO can exponentially estimate disturbances with zero second-order derivative but is subject to estimate error for disturbances with non-zero second-order derivative; iii) the third-order HODO can exponentially estimate disturbances with zero third-order derivative but is subject to estimate error for disturbances with non-zero third-order derivative; iv) by increasing the order of HODO, the HODO can also effectively estimate lowfrequency disturbance.

\section{B. Linear case: double-effect pilot plant evaporator}

In order to show the effectiveness of the proposed outputbased HODO for linear systems and its advantages over the traditional ESO [19] and generalized ESO [17, 18], the example from [26] is illustrated in this section. The plant is a double-effect pilot plant evaporator represented by a fifth- order linear model with the system matrices given by

$$
\begin{aligned}
A & =\left[\begin{array}{ccccc}
0 & 0 & -0.0034 & 0 & 0 \\
0 & -0.041 & 0.0013 & 0 & 0 \\
0 & 0 & -1.1471 & 0 & 0 \\
0 & 0 & -0.0036 & 0 & 0 \\
0 & 0.094 & 0.0057 & 0 & -0.051
\end{array}\right], \\
B & =\left[\begin{array}{ccc}
0 & 0 & 0 \\
0 & 0 & 0 \\
0 & 0 & 0.948 \\
0.916 & -1 & 0 \\
-0.598 & 0 & 0
\end{array}\right], D=\left[\begin{array}{cc}
0.062 & -0.132 \\
0 & -7.189 \\
0 & 0 \\
0 & 0
\end{array}\right], \\
C & =\left[\begin{array}{ccccc}
1 & 0 & 0 & 0 & 0 \\
0 & 1 & 0 & 0 & 0
\end{array}\right] .
\end{aligned}
$$

We can check that the pair $(A, C)$ is not observable, which means the traditional ESO [19] and full-order HODO [17, 18] can not be applied any more. However, if we select the functional matrix $L_{0}$ as

$$
L_{0}=\left[\begin{array}{lllll}
0 & 0 & 1 & 0 & 0
\end{array}\right],
$$

we can verify that the existence conditions for functional observer of $L \bar{x}$ in [25] are satisfied, which means the HODO in this paper is still applicable.

In the following simulation study, only first-order and second-order HODO are performed to illustrate the principle of the proposed HODO. Since we have formulated the HODO design problem into a special functional observer problem, the functional observer tool in [25] can be borrowed to facilitate our observer design. The poles of functional observer matrix $F$ for first-order HODO and second-order HODO are designed as $-3.1,-3.2,-3.3$ and $-3.1,-3.2,-3.3,-3.4,-3.5$, respectively.

The initial values of the system state are selected as $x_{0}=$ $[0,0,2,1,1]^{T}$, the initial values of the first-order and secondorder HODO are selected as zero vectors. The profile of disturbance $d_{1}$ is shown in the upper plot of Fig. 3, and $d_{2}$ is chosen as $d_{2}=-d_{1}$. The simulation results of first-order and second-order HODO for the estimate of $x_{3}$ and $d_{1}$ are shown in Figs. 2 and 3 respectively.

We can see from the Figs. 2 and 3 that the first-order HODO can asymptotically estimate both the state and disturbance for step disturbance but result in estimation error for ramp disturbance; while the second-order HODO can asymptotically estimate both the state and disturbance in the presence of both step disturbance and ramp disturbance. This means that the disturbance estimation performance can be improved by properly incorporating more disturbance model information.

\section{CONCLUSIONS}

This paper considers the problem of high order disturbance observer design for both linear and nonlinear system. The derivative and high order derivatives of disturbances are obtained by the proposed approach. We also establish the relationship of the proposed approach with the existing results. Finally, practical examples in the field of fault diagnosis 

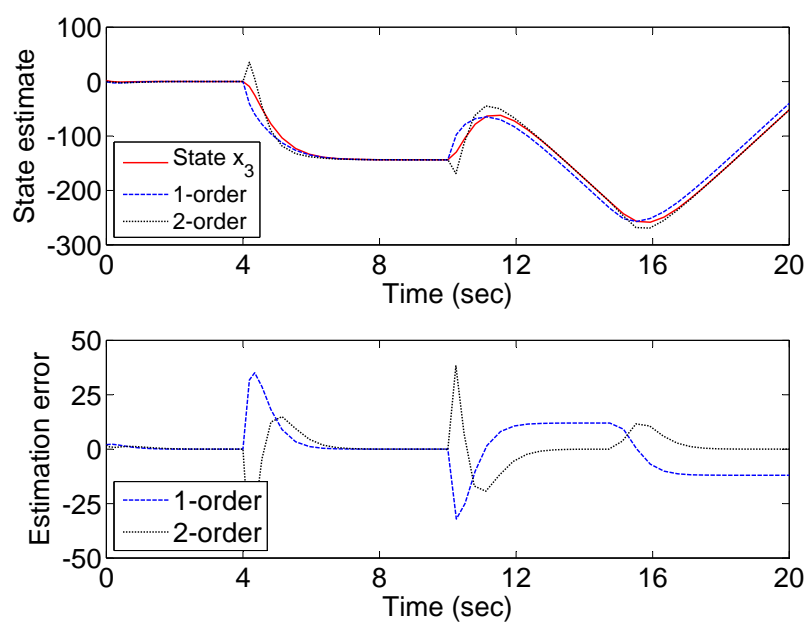

Fig. 2

$x_{3}$ ESTIMATE USING FIRST AND SECOND-ORDER HODOS
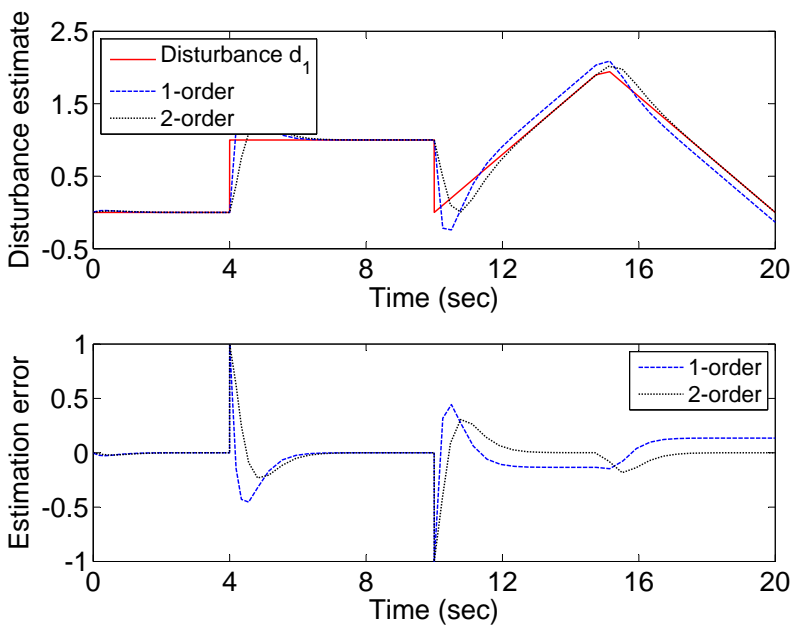

Fig. 3

$d_{1}$ ESTIMATE USING FIRST AND SECOND-ORDER HODOS

for a nonlinear missile system and disturbance estimation for a linear double-effect pilot plant evaporator systems with unobservable states are provided to illustrate the effectiveness of the proposed approach. The detailed discussion on the minimal-order output-based HODO design for linear systems will be provided in future study.

\section{REFERENCES}

[1] K. Ohishi, M. Nakao, K. Ohnishi, K. Miyachi, Microprocessorcontrolled DC motor for load-insensitive position servo system. IEEE Trans. Ind. Electro., (1), 44-49, 1987.

[2] W. Chen, D. J. Ballance, P. J. Gawthrop, J. O'Reilly, A nonlinear disturbance observer for robotic manipulators. IEEE Trans. Ind. Electro., 47(4), 932-938, 2000.

[3] W. Chen, Nonlinear disturbance observer-enhanced dynamic inversion control of missiles, J. Guid. Control Dynam., 26(1), 161-166, 2003.

[4] W. Chen, Disturbance observer based control for nonlinear systems. IEEE/ASME Trans. Mechatron., 9(4), 706-710, 2004.
[5] Li, S., Yang, J., Chen, W. H., \& Chen, X. (2014). Disturbance Observer-based Control: Methods and Applications. CRC Press.

[6] D. Koenig, Unknown input proportional multiple-integral observer design for linear descriptor systems: application to state and fault estimation. IEEE Trans. Autom. Control, 50(2), 212217, 2005.

[7] J. Su, W. H. Chen, B. Li, Disturbance observer based fault diagnosis. In 33rd Chinese Control Conference, 3024-3029, IEEE, 2014.

[8] J. Su, B. Li, W. Chen, On existence, optimality and asymptotic stability of the Kalman filter with partially observed inputs. Automatica, 53, 149-154, 2015.

[9] J. Su, B. Li, W. Chen, Recursive filter with partial knowledge on inputs and outputs. Int. J. Autom. Comput., 12(1), 35-42, 2015

[10] A. Isidori, Nonlinear Control Systems. Springer, Berlin, 1995.

[11] L. Guo, W. H. Chen, Disturbance attenuation and rejection for systems with nonlinearity via DOBC approach. Int J. Robust Nonlin., 15(3), 109-125, 2005.

[12] T. Umeno, Y. Hori, Robust speed control of DC servomotors using modern two degrees-of-freedom controller design. IEEE Trans. Ind. Electro., 38(5), 363-368, 1991.

[13] K. S. Kim, K. H. Rew, S. Kim, Disturbance observer for estimating higher order disturbances in time series expansion. IEEE Trans. Autom. Control, 55(8), 1905-1911, 2010.

[14] S. Phadke, P. Shendge, D. Ginoya, Sliding mode control for mismatched uncertain systems using an extended disturbance observer. IEEE Trans. Ind. Electro., 61(4), 1983-1992, 2014.

[15] S. Sadhu, T. K. Ghoshal, Sight line rate estimation in missile seeker using disturbance observer-based technique. IEEE Trans. Control Syst. Technol., 19(2), 449-454, 2011.

[16] G. P. Jiang, S. P. Wang, W. Z. Song, Design of observer with integrators for linear systems with unknown input disturbances. Electronics Letters, 36(13), 1168-1169, 2000.

[17] R. Miklosovic, A. Radke, Z. Gao, Discrete implementation and generalization of the extended state observer. In American Control Conference, 2209-2214, IEEE, 2006.

[18] A. Godbole, J. P. Kolhe, S. E. Talole, Performance analysis of generalized extended state observer in tackling sinusoidal disturbances, IEEE Trans. Control Syst. Technol., 21(6), 22122223, 2013

[19] J. Han, From PID to active disturbance rejection control. IEEE Trans. Ind. Electro., 56(3), 900-90. 2009.

[20] W. Kim, D. Shin, C. Chung, Microstepping using a disturbance observer and a variable structure controller for permanent-magnet stepper motors. IEEE Trans. Ind. Electro., 60(7), 2689-2699, 2013.

[21] H. Sun, S. Li, J. Yang, W. X. Zheng, Global output regulation for strictfeedback nonlinear systems with mismatched nonvanishing disturbances. Int J. Robust Nonlin., DOI: 10.1002/rnc.3216, 2014.

[22] J. Yang, S. Li, J. Su, X. Yu, Continuous nonsingular terminal sliding mode control for systems with mismatched disturbances. Automatica, 49(7), 2287-2291, 2013.

[23] J. Yang, J. Su, S. Li, X. Yu, High-order mismatched disturbance compensation for motion control systems via a continuous dynamic sliding-mode approach. IEEE Trans. Ind. Informat., 10(1), 604-614, 2014

[24] J. Su, J. Yang, S. Li, Continuous finite-time anti-disturbance control for a class of uncertain nonlinear system. Trans. I. Meas. Control, 26(3), 300-311, 2014.

[25] M. Darouach, Existence and design of functional observers for linear systems. IEEE Trans. Autom. Control, 45(5), 940-943, 2000.

[26] K. S. Kim, K. H., Rew, Reduced order disturbance observer for discrete-time linear systems. Automatica, 49(4), 968-975, 2013. 